\title{
2.4 METHODOLOGISCHE KONSEQUENZEN
}

\subsubsection{Sich selbst beforschen - Die Relevanz der Methode}

In Hinblick auf die methodologischen Konsequenzen einer Wissenschaftssoziologie der Soziologie haben wir bereits in Kapitel 2.3 gehört, dass die Untersuchung der Soziologie einigen Herausforderungen ausgesetzt ist:

1) Das Problem der doppelten Konstitution der Soziologie: Wie also kann man methodisch und methodologisch mit der "soziologischen Selbstreferenz“ (Burkart, 2003, S. 12) umgehen?

2) Als Einheimische unter Einheimischen sind besondere Bedingungen der eigenen Wissensproduktion zu beachten und zu reflektieren. Wie verändern die Untersuchenden ihr Untersuchungsobjekt (Breuer, 2003)?

3) Welche Methode ist geeignet, um Grenzziehungen in der Soziologie, die über Rhetorik und Semantik geschehen, zu reflektieren?

4) Auf welcher Ebene sollten diese Grenzziehungen betrachtet werden?

Die Reflexion dieser Aspekte ist in Hinblick auf eine geeignete Methodologie wie auch für die Auswahl einer Methode von besonderer Relevanz. Grundlegend wird die empirische Analyse der Soziologie von Soziolog(inn)en kritisch gesehen ${ }^{51}$, und sie beschränkt sich meist auf theoretische Aspekte (Breuer, 2003; Lau et al., 1989). Die empirische Untersuchung hingegen unterliegt nicht nur aufgrund der Zugehörigkeit der Forschenden zum Untersuchungsgegenstand selbst einem „Thematisierungstabu“ (das, wie wir bis jetzt erfahren haben, gilt ja nicht nur für diese Art von soziologischer Analyse), sondern auch wegen möglicher Kritik anderer Soziolog(inn)en (Burkart, 2003). Wenige haben bislang diesen Weg eingeschlagen. Wir haben im theoretischen Teil einige Vertreter(innen) einer selbstreflexiven und empirisch engagierten Soziologie der Soziologie kennengelernt. $\mathrm{Zu}$ den betreffenden Publikationen zählen der Sammelband von Camic et al. (2011) wie auch Bourdieus Analysen im „Homo Economicus“ (Bourdieu, 1984). Schelsky kritisierte als Anti-Soziologe seinerzeit das Fach mit am stärksten, indem er die intellektuelle Vorherrschaft der Soziologie neben anderen Intellektuellen als den ,uralten Widerstreit von weltlicher und geistlicher Herrschaft in einem modernen Gewande“ (Schelsky, 1975, S. 13) bezeichnete:

51 Diese Erfahrung durfte ich auch in zahlreichen Vorträgen zu meinem Forschungsthema machen. 
„Die Soziologenherrschaft kann nur von ,Soziologen“ bekämpft werden, also wenn man will, von ,ideellen Überläufern', genauso wie Absolutismus und Feudalismus im Adel (wie z.B. in Charles de Secondat, Baron de la Brede et de Montesquieu, oder in Claude Henry de Rouvroy, Graf von Saint-Simon, Enkel des Herzogs und Schriftstellers Louis de Rouvroy) und die Bourgeoisie in abtrünnigen Bürgern und Unternehmern wie Marx und Engels ihre treffsichersten Kritiker fand. Ohne den Anspruch ähnlicher Prominenz zu erheben, würde ich mich mit dieser Schrift ohne Zögern in die Reihe gegenwärtiger ,AntiSoziologen“ selbst einreihen.“(Schelsky, 1975, S. 259)

Die Perspektive, die diese Soziologen - unter Kritik ihrer Fachkollegen - versucht haben einzunehmen, war die eines distanzierten Blicks auf ihren Objektbereich. Dabei ist es jedoch essenziell, den geeigneten Umgang mit diesem Thema $\mathrm{zu}$ finden. Bourdieu (1984) tat dies, indem er letztlich seine Theorie der Praxis mit den dazugehörigen Begrifflichkeiten wie soziale Felder, Kapitalarten, illusio und Habitus auf das Wissenschaftssystem selbst anlegte. Schelsky hingegen löste mit seinen Schriften eine heftige Kontroverse aus, die Luhmann darauf zurückführte, dass Schelsky seine Thesen zu wenig wissenschaftlich reflektiert habe: Denn die Soziologie, zugehörig zum System Wissenschaft, hat Luhmann folgend nur diese Arbeitsgrundlage.

„Das mag der tragische Fall Helmut Schelskys belegen, den seine Enttäuschung mit dem Wirklichkeitssinn und dem gesellschaftlichen Verhalten von Soziologen dazu geführt hat, zuletzt als Antisoziologe aufzutreten, um vor der Soziologie zu warnen, gerade damit aber durchaus im Trend der sinkenden Reputation des Faches lag, und all dies reflektierte, aber nicht in eine dafür geeignete Form der Publikation bringen konnte. Was blieb, war Polemik.“(Luhmann, 1993, S. 252)

Diese Ausschnitte sollen verdeutlichen, wie wichtig es ist, eine Untersuchung der Soziologie theoretisch und methodologisch zu reflektieren. Was an Schelskys Vorgehen letztlich kritisiert wurde, war eine mangelnde Distanz zu den politischen Aktivitäten der Soziologinnen und Soziologen, die in einem polemischen Gewand daherkam. Wobei man natürlich auch gegenteilig argumentieren könnte, dass gerade Schelsky eine enorme Distanz zur Soziologie aufbringen musste, um eine derartige Kritik anzubringen. Egal, wie man es wendet, die Selbstreferenz der Soziologie erfordert unter methodologischen Gesichtspunkten eine besondere Reflexion. Breuer (2003) nennt vier Aspekte der Selbstreflexion im empirischen Prozess: 1. Die Standpunktgebundenheit des Wissens; 2. Die Kabinenhaftigkeit der Erkenntnis; 3. Die „Sinnesgebundenheit“, „Konzept-/Schema/Sprachgebundenheit“ und „Instrumentengebundenheit“" sowie 4. Die Interaktivi- 
tät zwischen Erkenntnissubjekt und -Objekt bzw. die Interventionshaftigkeit des epistemologischen Subjekt-Objekt-Kontaktes (Breuer, 2003, Sp. 4 ff.). Damit operationalisiert er Fragen, die bereits in der Systemtheorie wie auch in den STS reflektiert wurden. Unter „Kabinenhaftigkeit“ ist letztlich nichts anderes zu verstehen als das, was Luhmann mit dem „blinden Fleck des Beobachters“ bezeichnet, der sich der Beobachtung entzieht.

Die „Standpunktgebundenheit“ wie auch die „Konzept-/Schema-/Sprachgebundenheit" werfen das Problem auf, auf das die STS mit dem Prinzip der Inversion reagiert haben: Wir argumentieren immer aus einer bestimmten Perspektive heraus und sind auch in unseren kognitiven Prozessen an gewisse Formen gebunden. Die Schwierigkeit der Wissenschaftsforschung besteht ja genau darin, dass sie Prozesse der Wissensgenerierung kritisiert, denen sie letztlich auch selbst unterworfen ist. Deshalb soll laut STS mithilfe der reflexiven Ethnografie eine Außenposition konstruiert werden. Zudem plädieren die STS wie auch Luhmann dafür, eine eigene Sprache der Beschreibung zu finden (deren praktische Umsetzung jedoch nach wie vor nicht klar ist). Interaktivität und Interventionsfähigkeit stehen letztlich dafür, dass jegliche soziale Situation den Untersuchungsgegenstand verändert.

Zum einen geht es deshalb im Folgenden darum, eine Methode zu finden, die die Standpunktgebundenheit des Wissens reflektiert, eine Distanzierung vom Material zulässt und die Sprachgebundenheit einbezieht.

Hier orientieren wir uns einerseits an Karl Mannheim und der dokumentarischen Methode nach Bohnsack (1992; 1997a; 1993), andererseits an der Grounded Theory. Zunächst ist es mithilfe der dokumentarischen Methode möglich, diverse Perspektiven miteinander zu kontrastieren und sich selbst dem Text gegenüber fremd zu machen. Denn auch Karl Mannheim (1984; 2015[1929]) hat sich mit der Standpunktgebundenheit des Wissens auseinandergesetzt und die methodologischen Konsequenzen reflektiert. In Hinblick darauf, dass in dieser Arbeit eine „Einheimische“ ihren eigenen „Stamm“ untersucht, ist dies besonders wichtig. Die Grounded Theory, die eher als Methodologie denn als Methode verstanden werden kann, ist eine gute Ergänzung, da sie die Selbstreflexion im Forschungsprozess begünstigt. Sie hat ihre Wurzeln im Symbolischen Interaktionismus und arbeitet mit teilnehmender Beobachtung. Mithilfe von Forschungsmemos wird der Forschungsprozess im Einzelnen dokumentiert und reflektiert. Die Grounded Theory plädiert für eine Sensibilität, die den eigenen Einfluss auf das Forschungsumfeld berücksichtigt (siehe 3.1).

Natürlich kann keine Methode die oben genannten Herausforderungen gänzlich aus dem Weg räumen. Immer wird ein „blinder Fleck“ bleiben, denn auch wenn man unterschiedlichste Perspektiven einbezieht, können die Standortge- 
bundenheit des Wissens und die soziologische Selbstreferenz nicht einfach ausgelöscht werden. Mit der Reflexion der geeigneten Methodologie und der Auswahl der Methode nähert man sich jedoch zumindest einer Lösung der oben genannten Herausforderungen an.

\subsubsection{Semantiken und Rhetoriken als empirische Operationalisierung von Grenzarbeit}

Im Mittelpunkt der dokumentarischen Methode steht die Frage nach der unterschiedlichen Erzeugung und Zuschreibung von Sinn. Das Soziale beschränkt sich für Karl Mannheim nicht auf Strukturen, sondern besteht aus geistigen und kulturellen Gebilden. Menschen nehmen also, je nach Standortgebundenheit des Wissens, diverse Sinnzuschreibungen vor (Breuer, 2003, Sp. 15). Erfassbar werden diese Formen des Sinns dadurch, dass Mannheim zwischen 1. dem objektiven Sinn, 2. dem intendierten Ausdruckssinn und 3. der dokumentarischen Interpretation unterscheidet (Knoblauch, 2010, S. 105). Bohnsack (1997a) hat diesen Ansatz weiterentwickelt und fruchtbar gemacht: Sinnzuschreibungen werden in der dokumentarischen Methode nach Bohnsack überwiegend über hermeneutische Verfahren abgeleitet. Grundlegend ist dabei nicht nur, was gesagt wird, sondern vor allem, wie es gesagt wird.

Die dokumentarische Methode wird in dieser Arbeit konkret in der Analyse von Semantiken und Rhetoriken umgesetzt. Theoretisch haben wir bereits viel über Semantiken und Rhetoriken gehört. Beide erzeugen Sinn. Über eine hermeneutische Analyse sind sie als sinngebende Markierungen operationalisier- und erfassbar. Sie werden in der Theorie als Selbstbeschreibungen konkretisiert: In der Systemtheorie als Selbstbeschreibung des Systems, in den STS als ideologische Selbstbeschreibungen der Akteure. Hier lassen sich Konvergenzen zwischen Semantiken und Rhetoriken finden: Beide werden im Folgenden als hermeneutische Sinnkonstruktionen begriffen.

Greift man auf sprachliche Sinnstiftung zurück, hat das den Vorteil, wie bereits in den vorhergehenden Ausführungen angeklungen, dass man auf keinen bereits definierten Gesellschaftsbegriff oder andere strukturelle Aspekte der Grenzarbeit (Organisation, Profession) rekurrieren muss. Einem konstruktivistischen Paradigma entsprechend wird also nicht im Vorhinein definiert, was als Grenze gilt. Vielmehr wird aufgezeigt, wie diese hergestellt wird. Es soll also weniger um die Frage gehen, was das Problem eigentlich ist, als darum, wie das Problem konstruiert wird. Dadurch wird ein distanzierterer Blick auf das Material möglich. Es wird also, im Gegensatz zu soziologischen Betrachtungsweisen, weder auf ontologische noch auf autologische Schlüsse rekurriert, sondern es 
wird deren Konstruktionsform in den Blick genommen. Aus systemtheoretischer Sicht wird eine Beobachtungsposition zweiter Ordnung eingenommen.

\subsubsection{Akteure und ideologische Grenzarbeit}

Die Systemtheorie und die STS unterscheiden sich entscheidend in zwei Aspekten, die die Rolle von Rhetorik und Semantik angehen: 1. danach, wie Sinn konstruiert und eingesetzt wird; und 2. danach, ob dies auf der Ebene des Systems oder des Akteurs beobachtet wird. In den vorhergegangenen Ausführungen wurden bereits die Nachträglichkeit der Semantik wie auch die etwas starren Strukturen systemtheoretischer Selbstbeschreibungen reflektiert. Eine Analyse der Selbstbeschreibungen der Soziologie, die auf den Prämissen der Systemtheorie aufbaut, könnte nur auf der Ebene von Publikationen innerhalb des Wissenschaftssystems stattfinden. Kieserling $(2000 ; 2004)$ hat eine solche Untersuchung bereits in ausführlicher Weise für soziologische Selbstbeschreibungen vorgenommen. Berücksichtigt man jedoch die Annahme der STS, dass die Grenzen zwischen Wissenschaft und Nicht-Wissenschaft fluide sind und dass Rhetoriken strategisch eingesetzt werden, so bricht das glatte Bild der Selbstbeschreibungen auf und kann im Einzelnen auf der Akteurs- oder Mikroebene untersucht werden. Auch die Annahme Gieryns, dass solche ideologisch bedingten Selbstbeschreibungen ausschließlich auf Interessenkalkül aufbauen, sollte kritisch überprüft werden. Bezieht man zudem die Akteursebene ein, eröffnet sich in Hinblick auf Selbstbeschreibungen die Möglichkeit, wesentliche Fragen zu beantworten, die bereits Mulkay (1976) formulierte:

„How do scientists use vocabularies of justification inside their professional community? Are different vocabularies used in different social contexts; for example, in public as opposed to private media of communication? Are the more powerful groups and individuals better able to employ these vocabularies to serve their interests? With regard to the external relations of science, we are led to ask: How have scientists succeeded in gaining widespread acceptance of their ideology, if this is in fact the case? What range of political interests can be attributed to scientists and how exactly do such interests influence ideologies? Have scientists used different ideologies in ,non-democratic“ societies?“ (Mulkay, 1976, S. 654)

Konzentrieren wir uns auf die erste Frage und ergänzen wir sie um weitere Aspekte. Es geht also nicht nur darum, wie Wissenschaftler ,vocabularies of justification“ einsetzen. Wichtig ist auch, welche sprachlichen Bezüge sie überhaupt verwenden und welchen Stellenwert sie dieser Argumentation einräumen. Sind 
es, wie Luhmann oder auch Kieserling behaupten, rein selbstreferenzielle und soziologische Kategorien, die für eine Selbstbeschreibung angewandt werden? Oder sind noch andere Aspekte für eine Selbstbeschreibung relevant, die den Unterschied zwischen Soziologie und Nicht-Soziologie markieren? Wie unterscheiden sich diese Sinnangebote? Und sind diese Selbstbeschreibungen wirklich nur als „vocabularies of justification“ oder als Ideologie zu verstehen, oder stellen sie nicht vielleicht auch vocabularies of identification oder vocabularies of community building dar? Denn gerade in der Untersuchung von Grenzen zeigt sich nicht nur, wo eine Markierung gesetzt wird, sondern auch, inwieweit ähnliche oder differierende Grenzkonstruktionen einer Community bestehen und wie überhaupt diese Community selbst verstanden wird. Die zugrunde liegende These ist, dass Akteure innerhalb der Wissenschaft durch ihre Selbstbeschreibungen wiederum den Diskurs und die Systemgrenzen beeinflussen, also die Semantiken, die Soziologie von Nicht-Soziologie unterscheiden. Grenzen werden in alltäglichen Aushandlungen immer neu definiert und festgelegt. Dementsprechend können sie auch - in Anlehnung an Bourdieu und Giddens - systemverändernd wirken. 
